\title{
TRPC3 and TRPC6 Contribute to the Pathogenesis of Hypertension
}

\author{
Qin Zou', Cui Zhang1*, Yuqing Guo ${ }^{*}$ \\ ${ }^{1}$ Department of Microbiology and Immunology, Guangdong Pharmaceutical University, Guangzhou, China \\ ${ }^{2}$ Guangdong Pharmaceutical University, First Affiliated Hospital, Guangzhou, China \\ Email: ccuizhang@126.com, ${ }^{*}$ 532007099@qq.com
}

Received 24 August 2015; accepted 26 October 2015; published 29 October 2015

Copyright (C) 2015 by authors and Scientific Research Publishing Inc.

This work is licensed under the Creative Commons Attribution International License (CC BY).

http://creativecommons.org/licenses/by/4.0/

c) (i) Open Access

\begin{abstract}
Recently studies found that TRPC3 and TRPC6 played an important role in cardiovascular disease. Hypertension, as a cardiovascular disease causing the highest morbidity and mortality, has close relationship with the expressions of TRPC3 and TRPC6. Unbalanced calcium homeostasis is the major factor of pathogenesis of hypertension. Changes of intracellular calcium concentration depend on calcium transmembrane transportation, intracellular calcium store releasing and other processes. TRPC3, TRPC6 molecules, as non-selective cation channels on the cell membranes, are involved in the processes. This review illustrated the functions of TRPC3 and TRPC6 on myocardial cells, smooth muscle cells and inflammatory cells in the development of hypertension, and the effects of drugs like sildenafil to provide a new way for the prevention and treatment of hypertension.
\end{abstract}

\section{Keywords}

TRPC3, TRPC6, Hypertension, Calcium

\section{Introduction}

Hypertension, as a cardiovascular disease causing the highest morbidity and mortality, is a kind of clinical syndrome performing characterized by a sustained high blood pressure, and belongs to the cardiovascular diseases. It can be divided into two categories: essential hypertension (EH, or primary systemic arterial hypertension) and secondary hypertension. The etiopathogenesis of essential hypertension is unclear, while the latter is caused by other diseases, which we know clearly, and its treatment is simpler than the former's. Increasing evidence supports a role for the transient receptor potential canonical 3, 6 (TRPC3, 6) channels in this pathophysiology.

*Corresponding author. 
TRPC3 and -6 channels belong to the seven TRPC sub-families of non-selective and non-voltage-gated ion channels that are activated by a phospholipase C (PLC) to control transmembrane calcium flux. TRPC3 channel is composed of 836 amino acids, while TRPC6 channel includes 931 amino acids. They form a structural and functional subfamily sharing $70 \%$ to $80 \%$ homology at the amino acid level [1]. And they are both characterized by six transmembrane domains with the pore-forming region between the fifth and sixth transmembrane domains, which is necessary for the production of a functional channel. Their N-terminal and C-terminal sequence are both in cell membrane [2]. The structures of TRPC3 and -6 channels are shown in Figure 1 and Figure 2.

TRPC 3 and -6 are often upregulated in cardiovascular disease, and their inhibitions ameliorate the associated pathophysiology. Changes of intracellular calcium concentration depend on calcium transmembrane transportation, intracellular calcium store releasing and other processes. TRPC3 and -6 molecules, as non-selective cation channels on the cell membrane, are involved in the processes. There are three kinds of channel to regulate the levels of intracellular calcium: voltage dependent calcium channel (VDCC), receptor operated calcium channel (ROCC) and store-operated calcium channel (SOCC). VDCC and ROCC flow a large amount of calcium in a short time, while SOCC produces small, continuous calcium flux. VDCC is not sensitive to stimulation and calcium depolarization blocker which differs from ROCC. TRPC3 and -6 both have dual characteristics of ROCC and SOCC [5]: on the one hand, they can be activated by diacylglycerol (DAG) upon stimulation of PLC-

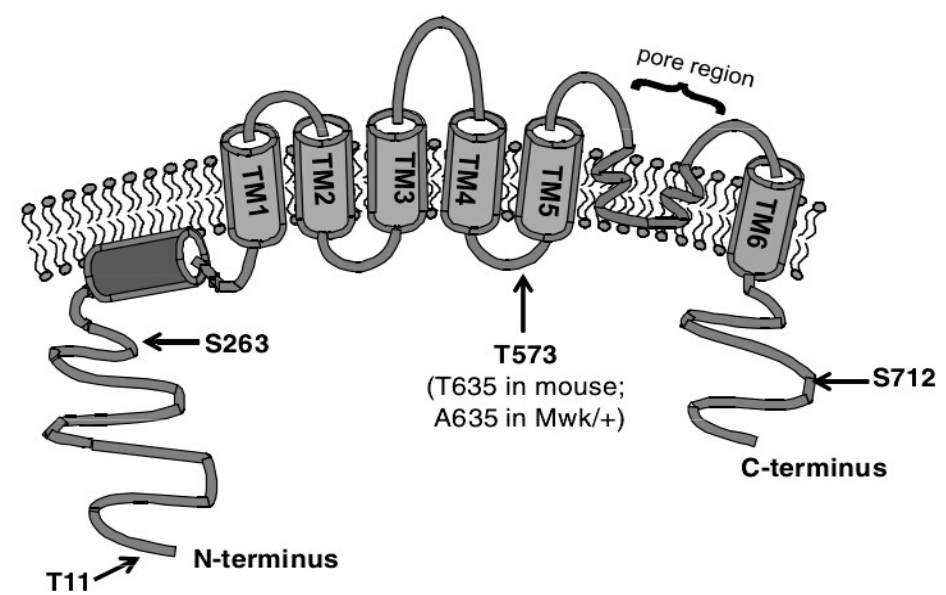

Figure 1. The structure of TRPC3 channel [3]. TRPC3 have cytoplasmic N- and C-termini, six transmembrane regions (TM), a pore region (between TM5 and TM6), a hydrophobic segment which does not seem to span the plasma membrane, and four ankyrin-like repeats in the cytoplasmic N-terminal region. Locations of different residues shown to be phosphorylated by PKC (Serine 712; S712) and PKG (Threonine 11; T11 and Serine 263; S263) on human TRPC3 are shown. Threonine 573 (T573) is the human equivalent of Threonine 635 (T635) in mouse TRPC3.

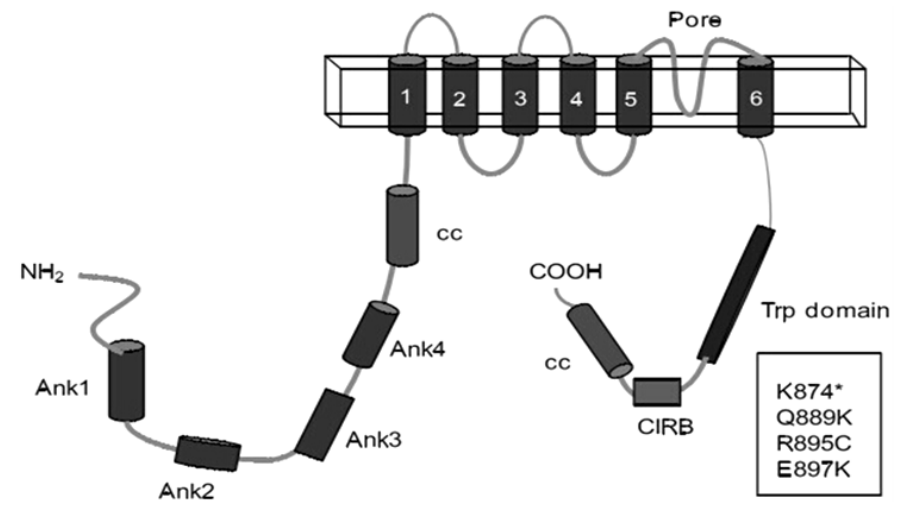

Figure 2. The structure of TRPC6 channel [4]. TRPC6 have cytoplasmic N- and C-termini, six transmembrane regions, a pore which is necessary for the production of a functional channel and a hydrophobic segment which does not seem to span the plasma membrane. Boxes indicate human mutations known to cause focal segmental glomerulosclerosis. All of the known disease-causing mutations are located in the cytoplasmic $\mathrm{NH}_{2}$ and $\mathrm{COOH}$ terminals of TRPC6. 
coupled receptors resulting in the breakdown of phosphoinositides. On the other hand, they are second messenger-operated calcium entry channels that can be activated by inositol 1, 4, 5-triphosphate to release the intracellular calcium, as is shown in Figure 3. This depletion can activate TRPC3 and -6 channels, namely SOCC features. The characteristics these two molecules playing depend on their expression level. When the expression decreased, TRPC3 and -6 are SOCC, or they are ROCC.

In addition, TRPC3 and -6 may through other signal molecules to regulate calcium concentration. TRPC3 mediates $\mathrm{Ca}^{2+}$ and $\mathrm{Na}^{+}$entry in proximity of $\mathrm{Na}^{+} / \mathrm{Ca}^{2+}$ exchanger (NCX), thereby elevating cellular calcium levels and contractility. Excessive activation of TRPC3 is associated with transient cellular calcium overloading, spatial uncoupling between TRPC3 and NCX [6]. While according to new research in human glomerular mesangial cells, calcium sensing receptors (CaSR) can be adjusted by raising the expression of TRPC3 and -6 to flow calcium [7]. The signal pathways of TRPC3, 6 are shown in Figure 3.

Recent studies indicate that TRPC3, 6-mediated cellular calcium homeostasis plays an important role in the pathogenesis of hypertension. We review the literature on TRPC3 and -6 in different cells, including the myocardial cells, smooth muscle cells and inflammatory cells, and effects of drugs like sildenafil, which may provide a novel therapeutic approach for treatment of hypertension.

\section{TRPC3, 6 Contribute to Hypertension}

TRPC3 and -6 regulate intracellular calcium homeostasis, which is involved in hypertension. Therefore, the studies about patients and animal models with hypertension disease have shown that TRPC3, 6 expression would promote the occurrence of hypertension and applying corresponding inhibitor could improve the symptoms of hypertension.

\subsection{TRPC3, 6 in SHR}

Hypertension occurring on animal models mainly includes spontaneous hypertension, induced hypertension and genetic engineering hypertension, in which spontaneous hypertension animal models called spontaneous hypertension rat (SHR). SHR was produced from Wistar rats' selective mating by Okamoto in 1963. The key feature of SHR is their high hypertension incidence without obvious primary kidney or adrenal injury. Their lifetime is long, which can live 13 - 14 months [8]. SHR is internationally recognized as the most close to human essential hypertension animal model, and has been widely used in the basic research of primary hypertension and antihypertensive drug studies.

In 2005, it was found that increased TRPC3 channel expression and increased TRPC3-related calcium influx in SHR for the first time [9]. Then Chen et al. [10] found that TRPC3 increased in SHR carotid artery and mesenteric artery. The expression of TRPC6 can also affect the incidence of hypertension. Wu [11] showed that in SHR left ventricular TRPC6 expression level was higher than control group with the same weeks. Upregulation

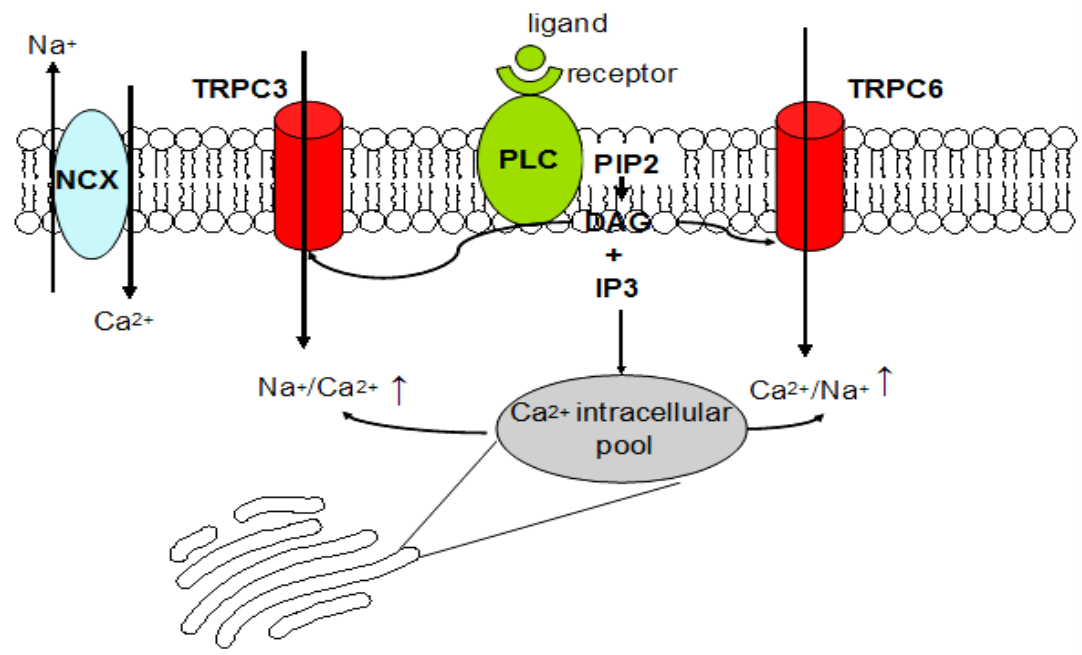

Figure 3. Signal pathway of TRPC3, 6 at the plasma membrane (modified from reference [5] [6]). 
of TRPC3 and TRPC6 may be involved in carotid arterial remodeling in SHR [12], which contribute to the development of pulmonary arterial hypertension. In the aorta of SHR, the increased expressions of TRPC3 and -6 are closely associated with elevated blood pressure variability and might be involved in the regulation of blood pressure variability [13]. All the animal models experiment above confirmed that rise in TRPC3, -6 protein expression is closely related to the development of hypertension.

\subsection{TRPC3, 6 in Essential Hypertension}

In the studies of clinical patients with hypertension, expressions of TRPC3 and -6 are also associated with hypertension. Initially, Liu D. [14] observed a significant increase of TRPC3 protein expression in essential hypertensive patients compared with normotensive control subjects, which increase the strength of the calcium influx to aggravate the condition of hypertensive patients. Further research showed the monocyte migration function of patients was associated with an increased expression of TRPC3, while inhibiting TRPC3 molecules could prevent mononuclear cells after migration [15]. Compared TRPC3, -6 expression level in the monocytes of patients with primary hypertension and normal people, Liu and his teammates [16] once again confirmed that the mononuclear cells in patients with TRPC3 expression was increased, but TRPC6 expression had no change. The functions of TRPC3 and -6 in hypertension aren't same, which is still unknown and remains to be elucidated.

The roles of TRPC3 and -6 in the mechanism of essential hypertension are associated with regulation of intracellular calcium concentration, which are important factors to hypertension development. Increases of intracellular calcium induce cellular reaction including cell growth, differentiation, contraction and secretion, which is initiated by the activation of phospholipase C. It is activated by receptor stimulation, causing the hydrolysis of phosphatidylinositol-4, 5-bisphosphate (PIP2) and generating two second messengers, IP3 and DAG. IP3 binds to its specific receptor-channel on the endoplasmic reticulum and causes the calcium mobilization by releasing calcium from the intracellular pool. In the second phase, calcium enters through the plasma membrane and no cellular activity has ever been observed in the absence of this second phase of calcium mobilization. TRPC3 and -6 are involved in calcium entry into cells upon hormonal stimulation to make the rise of proliferation of myocardial cells and smooth muscle cells (SMC) and SMC contraction. Finally increasing peripheral resistance aggravates the pathological process of high blood pressure [17]-[19]. Therefore, TRPC3 and -6 expression disorders can mediate calcium balance contributing the formation of high blood pressure.

\subsection{TRPC3, 6 and Different Cell Types in Hypertension}

The influence of TRPC3, TRPC6 on hypertension mainly relates to myocardial cells, smooth muscle cells and inflammatory cells.

\subsubsection{Effects of TRPC3, 6 on Myocytes}

Cardiomyocytes and myocardial fibroblasts account for over $90 \%$ of the heart tissue cells. The changes of myocardial cell and myocardial fibroblasts proliferation are important promoting factors of hypertensive myocardial hypertrophy, which linked the change of intracellular calcium concentration closely. In myocardial cells calcium influx is mainly affected by VDCC, which TRPC3 and -6 molecules influence the change of myocardial intracellular calcium to form hypertension.

Research has shown that mice with heart-specific overexpression of TRPC6 developed spontaneous cardiac hypertrophy and remodeling [20]. At the same time, in mild hypoxia-induced cardiomyocytes hypertrophy, TRPC3 and -6 were increased, and calcium-calcineurin signals were also enhanced in a time-dependent manner [21]. Recently study showed that in adult feline myocytes, TRPC3, 6 over expression could induce hypertrophic signaling [22]. In cardiac fibroblasts, we saw the same. Masahide and colleagues [23] demonstrated that TRPC3 was highly expressed in freshly isolated fibroblasts. TRPC6 overexpression promotes fibroblast to myofibroblast conversion [24]. Visibly, the expression of TRPC3 and -6 molecules in the process of hypertensive pathology is abnormal, which caused by the imbalance of myocardial cell proliferation.

Changes of hypertensive myocardial cells and cardiac fibroblasts associated with unbalanced calcium homeostasis, which caused TRPC3 and -6 overexpression leading to calcineurin-NFAT signal pathways dysregulating. NFAT means nuclear factor of activated T cells which is the most important of calcineurin substrates. In 2006 studies found that TRPC3 could activate NFAT signal path for the first time [25]. And Koichiro and his 
workmates [26] found that TRPC6 also regulated calcineurin-NFAT signaling and was a key component of a calcium-dependent regulatory loop that drives pathologic cardiac remodeling. TRPC3 and -6 activation and inward calcium entry stimulate calcineurin, which in turn de-phosphorylates NFAT leading to the nuclear translocation of the latter. As the promoter region for TRPC3 and -6 contains NFAT-binding sites, this provides a positive-feedback mechanism to further upregulate channel expression and activity. Intracellular calcium concentration increased when the intracellular calcium level was too high. Then the myocardial cells shrink, and there were excessive proliferation and increasing peripheral resistance, which increased blood pressure. However, if there are other signal transduction mechanisms in the process of participation still need further in-depth study.

\subsubsection{Effects of TRPC3, 6 on SMC}

Functions of SMC including contraction, relaxation and proliferation, of which abnormal changes can make peripheral vascular resistance increasing to form hypertension [27]. In SMCs, overexpression of TRPC3 and -6 will make abnormal cell proliferation affecting the development of hypertension. In SHR model of carotid artery SMCs, the increased expression of TRPC3 may lead to polarization of smooth muscle and increasing vascular contractility [28]. TRPC6 expression also increased in pulmonary vascular SMCs, which is essential to hypoxic pulmonary hypertension formation factors [29]. The inhibition of TRPC6 expression decreased the intracellular calcium concentration improving the SMC proliferation to control blood pressure [30]. Therefore, changes of TRPC3 and -6 in SMCs affect the formation of hypertension.

The impact of TRPC3 and -6 on SMC is also related to oxygen concentration. In 2004, study had showed that for the first time TRPC3 and -6 channels of pulmonary arterial SMCs were upregulated by chronic hypoxia and contributed to the enhanced vascular tone in hypoxic pulmonary hypertension [31]. In mild hypoxia-induced cardiomyocytes hypertrophy, TRPC3 and -6 up-regulations and enhanced calcium-calcineurin signals were inhibited by a hypoxia-inducible factor 1(HIF-1) specific blocker, whereas promoted by HIF-1 $\alpha$ overexpression. Hypoxia triggered intracellular calcium contributes to promoted proliferation and migration of SMCs then make the vasoconstriction to increase blood pressure [32].

Recent studies also found that TRPC3 and -6 participated in the proliferation of pulmonary artery smooth muscle cells induced by the endothelin-1 (ET-1) [33]. And TRPC3 also participated in the calcium influx in induced by angiotensin II (Ang II) [34]. Ang II and ET-1 are both associated with hypertension microvascular reconstruction. TRPC3 and -6 may through participation in Ang II, ET-1 and DAG of signaling pathways that affect the SMC leading to the change of blood pressure, but the specific mechanism of action.

\subsubsection{Effects of TRPC3, 6 on Inflammatory Cells}

Inflammatory cell is a kind of cell that takes part in inflammation, which is a significant regulator of hypertension [35]. It includes monocytes, macrophage, lymphocytes, mast cells, fibroblast and so on. TRPC3 and -6 have effect on these inflammatory cells. Zhao et al. [14] demonstrated that TRPC3 contributed to the increased migration of monocytes in essential hypertension. In studies aimed at characterizing the response of human monocytes exposed to high glucose and oxidative stress, protein levels of TRPC3 and -6 were found to be increased [36], and TRPC6 mRNA was also augmented in circulating monocytes from patients with type 2 diabetes [37]. TRPC3 and -6 channels also contribute to degranulation in FcepsilonRI-stimulated mast cells [38].

Lymphocytes are believed to use SOCC as the main mode of calcium influx [39] and TRPC3, 6 can be activated by DAG as SOCC. Direct involvement of the TRPCs in SOCC remains controversial with no conclusive reports of store-operated TRPC3, 6 calcium currents in lymphocytes. The evidence surrounding the function of TRPC3, 6 channels in SOCC have been discussed.

Whereas none of these studies explored whether increased TRPC3, 6 levels had a direct impact on signaling events associated to inflammatory cell, the evidence provided is suggestive of a potential novel link between the expression or function of TRPC3, 6 and inflammatory cell's role in vascular disease associated to hypertension.

\section{TRPC3, 6 Inhibition as a Therapeutic}

There are a lot of drugs treating hypertension disease, but medicine aiming at the expression of TRPC3 and -6 isn't too much. We can inhibit TRPC3 and -6 to improve the development of hypertension disease. Their inhibitors conclude SKF96365, an inhibitor of TRPC not specific for any TRPC subtype, Pyr3, a selective inhibitor for TRPC3 and other small-molecule inhibitors. Sildenafil and some antihypertensive drugs also can affect 
TRPC3, 6 expressions.

\subsection{Small-Molecule Inhibitors of TRPC3, 6}

There are a number of small-molecule inhibitors such as SKF96365 and Pyr3. SKF96365 has been used experimentally to hamper the current of TRPC channels [40], and it is nonselective. Pyr3 has been identified as a selective TRPC3 antagonist and can diminish hypertrophic signaling in pressure-overloaded hearts in vivo [41]. Selective small-molecule inhibitors remain scarce, and none target both TRPC3 and -6 channels, which may be useful given the high homology among them and evidence of redundant signaling. Lately researchers tested selective TRPC3, 6 antagonists (GSK2332255B and GSK2833503A) and found dose-dependent blockade of cell hypertrophy signaling triggered by Ang II or ET-1 in cardiac myocytes [42]. Intriguingly, although gene deletion of TRPC3 or -6 alone did not protect against pressure overload, combined deletion was protective, supporting the value of dual inhibition. Further development of this pharmaceutical class may yield a useful therapeutic agent for heart disease management.

\subsection{Effect of Sildenafil on the Expression of TRPC3, 6}

Sildenafil, a potent phosphodiesterase type 5 (PDE5) inhibitor, has been proposed as pulmonary arterial hypertension (PAH) agents. In the pulmonary artery smooth muscle cells of patients with $\mathrm{PAH}$, the changes of intracellular calcium concentration are regulated by TRPC3 and -6 channels. Koitabashi [43] for the first time reported that sildenafil could block TRPC6 gene and protein expression, which was dependent on the activation of protein kinase G (PKG) and participating in regulating NFAT signal path. Kiso [44] found that in rat myocardial cells, through ET-1 stimulate with sildenafil combination therapy, sildenafil inhibits cardiomyocyte hypertrophy by suppressing the up-regulation of TRPC3 and -6 expressions to treat pulmonary hypertension. Wang et al. [45] used sildenafil to regulate the expression of TRPC6 in the pulmonary artery smooth muscle cells, and the results showed that the drug was dependent on cGMP-PKG signaling pathways to control TRPC6 expression. Thus restraining the calcium inflow plays a role of regulation in pulmonary arterial hypertension.

Sildenafil in the treatment of pulmonary hypertension may be through the cGMP-PKG signaling pathways to regulate TRPC3 and -6 molecules: sildenafil as a potent PDE5 inhibitor can block the PDE decomposing cGMP to activate PKG, then make TRPC3 and -6 phosphorylated and lose activity. Extracellular calcium internal flow reduced so the blood pressure returned to normal level.

\subsection{Effect of Antihypertensive Drugs on the Expression of TRPC3, 6}

Common antihypertensive drugs include angiotensin converting enzyme inhibitors (ACEI), angiotensin receptor blockers (ARB) and calcium channel blockers (CCB) which means L-type voltage dependent calcium channel (L-VDCC) blockers. These three kinds of drugs can improve the symptoms of hypertension. They are respectively represented by ramipril, valsartan and amlodipine. Related researchers mainly focused on the influence of these drugs on expressions of TRPC3 and -6 in hypertension.

After using ramipril and valsartan, Liao and his workmates [46] found that the two could reduce expression of TRPC3 protein in aortic smooth muscle cells of SHR. Other results suggest that in SHR myocardial cells, these drugs can significantly reduce expressions of TRPC3 and -6 [47], which can prevent Ang II combining with angiotensin 1 receptor to block signaling pathways. Expressions of TRPC6 increased in Ang II-induced hypertension mice, which prompted that Ang II associated with the expression of TRPC6 [48]. Naoya et al. [49] proved that DAG-induced calcium signaling pathway through TRPC3 and -6 was essential for Ang II-induced NFAT activation. Further studies confirmed that the effects of Ang II and its pathogenic role in hypertension disease involved enhanced TRPC6 expression via a calcineurin-NFAT positive feedback signaling pathway [50]. Researchs above show that the expressions of TRPC3 and -6 are regulated by Ang II, of which mechanism may be: Ang II combined with angiotensin 1 receptor, which activated PLC-calcineurin-NFAT signal pathway, thus raising TRPC3 and -6 expressions and causing high blood pressure.

Using amlodipine to treat hypertension almost has no effect on TRPC3 and -6 expressions. It is perhaps that these drugs belong to the ROCC rather than VDCC, while L-VDCC channel blocker amlodipine can't inhibit TRPC3, 6 expression. But there is still a research shows that amlodipine can slightly inhibit TRPC3 and -6 proteins. Their specific regulatory mechanism has yet to be further studied. 


\section{Conclusion and Prospects}

Through the comprehensive analysis, it is clear that TRPC3 and -6 are important in the formation of high blood pressure, resulting from the disorder expression and intracellular calcium homeostasis unbalance. People also make attention to the change of drug action and the differences and similarities which may provide a new route for treating disease if the TRPC3 and -6 channels are regarded as molecular targeted therapy. These studies provide a new perspective on the pathogenesis of cardiomyopathies and open new avenues for treatment of hypertension disease.

\section{Acknowledgements}

This work was supported by the Joint Natural Science Fostering Foundation of Guangdong Pharmaceutical University and The First Affiliated Hospital (Grant No.GYFYLH201319) and Supporting Program of South China Traditional Chinese Medicine City in Guangdong Province (Grant No.20101H014).

\section{References}

[1] Dietrich, A., Kalwa, H. and Gudermann, T. (2010) TRPC Channels in Vascular Cell Function. Thrombosis and Haemostasis, 103, 262-270. http://dx.doi.org/10.1160/TH09-08-0517

[2] Rowell, J., Koitabashi, N. and Kass, D.A. (2010) TRP-ing up Heart and Vessels: Canonical Transient Receptor Potential Channels and Cardiovascular Disease. Journal of Cardiovascular Translational Research, 5, 516-524. http://dx.doi.org/10.1007/s12265-010-9208-4

[3] Trebak, M. (2010) The Puzzling Role of TRPC3 Channels in Motor Coordination. Pflügers Archiv, 459, 369-375. http://dx.doi.org/10.1007/s00424-009-0740-5

[4] Dryer, S.E. and Reiser, J. (2010) TRPC6 Channels and Their Binding Partners in Podocytes: Role in Glomerular Filtration and Pathophysiology. American Journal of Physiology, 299, F689-701. http://dx.doi.org/10.1152/ajprenal.00298.2010

[5] Yuan, J.P., Kim, M.S., Zeng, W., Shin, D.M., Huang, G. and Worley, P.F. (2009) TRPC Channels as STIM1-Regulated SOCs. Channels (Austin), 3, 221-225. http://dx.doi.org/10.4161/chan.3.4.9198

[6] Doleschal, B., Primessnig, U., Wölkart, G., Wolf, S., Schernthaner, M., Lichtenegger, M., et al. (2015) TRPC3 Contributes to Regulation of Cardiac Contractility and Arrhythmogenesis by Dynamic Interaction with NCX1. Cardiovascular Research, 106, 163-173. http://dx.doi.org/10.1093/cvr/cvv022

[7] Meng, K., Xu, J., Zhang, C., Zhang, R., Yang, H., Liao, C. and Jiao, J. (2014) Calcium Sensing Receptor Modulates Extracellular Calcium Entry and Proliferation via TRPC3/6 Channels in Cultured Human Mesangial Cells. PLoS One, 9, e98777. http://dx.doi.org/10.1371/journal.pone.0098777

[8] Okamoto, K. and Aoki, K. (1963) Development of a Strain of Spontaneously Hypertensive rats. Japanese Circulation Journal, 27, 282-293.

[9] Liu, D., Scholze, A., Zhu, Z., Kreutz, R., Wehland-von-Trebra, M., Zidek, W., et al. (2005) Increased Transient Receptor Potential Channel TRPC3 Expression in Spontaneously Hypertensive rats. American Journal of Hypertension, 18, 1503-1507. http://dx.doi.org/10.1016/j.amjhyper.2005.05.033

[10] Chen, X.P., Yang, D.C., Ma, S.T., He, H., Luo, Z. and Feng, X. (2010) Increased Rhythmicity in Hypertensive Arterial Smooth Muscle Is Linked to Transient Receptor Potential Canonical Channels. Journal of Cellular and Molecular Medicine, 14, 2483-2494. http://dx.doi.org/10.1111/j.1582-4934.2009.00890.x

[11] Wu, Y. (2013) The Effect of TRPC6 on Left Ventricular Hypertrophy in SHR-The Study of the Relationship between TRPC6 and BNP. Fujian Medical University, Fuzhou.

[12] Lin, X.H., Hong, H.S., Zou, G.R. and Chen, L.L. (2015) Upregulation of TRPC1/6 May Be Involved in Arterial Remodeling in Rat. Journal of Surgical Research, 195, 334-343. http://dx.doi.org/10.1016/j.jss.2014.12.047

[13] Wang, J., Chen, Y., Lin, C., Jia, J., Tian, L. and Yang, K. (2014) Effects of Chronic Exposure to Cigarette Smoke on Canonical Transient Receptor Potential Expression in Rat Pulmonary Arterial Smooth Muscle. AJP: Cell Physiology, 306, C364-C373. http://dx.doi.org/10.1152/ajpcell.00048.2013

[14] Liu, D., Scholze, A., Zhu, Z., Krueger, K., Thilo, F. and Burkert, A. (2006) Transient Receptor Potential Channels in Essential Hypertension. Hypertension, 24, 1105-1114. http://dx.doi.org/10.1097/01.hjh.0000226201.73065.14

[15] Zhao, Z., Ni, Y., Chen, J., Zhong, J., Yu, H. and Xu, X. (2012) Increased Migration of Monocytes in Essential Hypertension Is Associated with Increased Transient Receptor Potential Channel Canonical Type 3 Channels. PLoS ONE, 7, e32628. http://dx.doi.org/10.1371/journal.pone.0032628 
[16] Liu, D.Y., Thilo, F., Scholze, A., Wittstock, A., Zhao, Z.G. and Harteneck, C. (2007) Increased Store-Operated and 1-Oleoyl-2-acetyl-sn-glycerol-Induced Calcium Influx in Monocytes Is Mediated by Transient Receptor Potential Canonical Channels in Human Essential Hypertension. Journal of Hypertension, 25, 799-808. http://dx.doi.org/10.1097/HJH.0b013e32803cae2b

[17] Imai, Y., Itsuki, K., Okamura, Y., Inoue, R. and Mori, M.X. (2012) A Self-Limiting Regulation of VasoconstrictorActivated TRPC3/C6/C7 Channels Coupled to PI(4,5)P2-Diacylglycerol Signaling. The Journal of Physiology, 590, 1101-1119. http://dx.doi.org/10.1113/jphysiol.2011.221358

[18] Itsuki, K., Imai, Y., Hase, H., Okamura, Y., Inoue, R. and Mori, M.X. (2014) PLC-Mediated PI(4,5) P2 Hydrolysis Regulates Activation and Inactivation of TRPC6/7 Channels. The Journal of General Physiology, 143, 183-201. http://dx.doi.org/10.1085/jgp.201311033

[19] Adebiyi, A., Thomas-Gatewood, C.M., Leo, M.D., Kidd, M.W., Neeb, Z.P. and Jaggar, J.H. (2012) An Elevation in Physical Coupling of Type 1 IP3 Receptors to TRPC3 Channels Constricts Mesenteric Arteries in Genetic Hypertension. Hypertension, 60, 1213-1219. http://dx.doi.org/10.1161/HYPERTENSIONAHA.112.198820

[20] Xie, J., Cha, S.K., An, S.W., Kuro-O, M., Birnbaumer, L. and Huang, C.L. (2012) Cardioprotection by Klotho through Downregulation of TRPC6 Channels in the Mouse Heart. Nature Communications, 3, 1238. http://dx.doi.org/10.1038/ncomms2240

[21] Chu, W., Wan, L., Zhao, D., Qu, X., Cai, F. and Huo, R. (2012) Mild Hypoxia-Induced Cardiomyocyte Hypertrophy via Up-Regulation of HIF-1 $\alpha$-Mediated TRPC Signaling. Journal of Cellular and Molecular Medicine, 16, 2022-2034. http://dx.doi.org/10.1111/j.1582-4934.2011.01497.x

[22] Catherine, A.M., Hong, Y.Z., Jennifer, D., Robert, N.C., Danielle, M.T., Nicholas, E.H., et al. (2014) Transient Receptor Potential Channels Contribute to Pathological Structural and Functional Remodeling after Myocardial Infarction. Circulation Research, 115, 567-580. http://dx.doi.org/10.1161/CIRCRESAHA.115.303831

[23] Yue, Z., Zhang, Y., Xie, J., Jiang, J. and Yue, L. (2013) Transient Receptor Potential (TRP) Channels and Cardiac Fibrosis. Current Topics in Medicinal Chemistry, 13, 270-282. http://dx.doi.org/10.2174/1568026611313030005

[24] Davis, J., Burr, A.R., Davis, G.F., Birnbaumer, L. and Molkentin, J.D. (2012) A TRPC6-Dependent Pathway for Myofibroblast Transdifferentiation and Wound Healing in Vivo. Developmental Cell, 23, 705-715. http://dx.doi.org/10.1016/j.devcel.2012.08.017

[25] Nakayama, H., Wilkin, B.J., Bodi, I. and Molkentin, J.D. (2006) Calcineurin-Dependent Cardiomyopathy Is Activated by TRPC in the Adult Mouse Heart. The FASEB Journal, 20, 1660-1670. http://dx.doi.org/10.1096/fj.05-5560com

[26] Koichiro, K., Yanggan, W., John, M.A., James, A.R., Rhonda, B.D., Joseph, A.H., et al. (2006) TRPC6 Fulfills a Calcineurin Signaling Circuit during Pathologic Cardiac Remodeling. Journal of Clinical Investigation, 116, 3114-3126. http://dx.doi.org/10.1172/JCI27702

[27] Goulopoulou, S. and Webb, R.C. (2014) Symphony of Vascular Contraction: How Smooth Muscle Cells Lose Harmony to Signal Increased Vascular Resistance in Hypertension. Hypertension, 63, e33-e39. http://dx.doi.org/10.1161/HYPERTENSIONAHA.113.02444

[28] Noorani, M.M., Noel, R.C. and Marrelli, S.P. (2011) Up-Regulated TRPC3 and Down-Regulated TRPC1 Channel Expression during Hypertension Is Associated with Increased Vascular Contractility in Rat. Frontiers in Physiology, 2, 1 8. http://dx.doi.org/10.3389/fphys.2011.00042

[29] Xia, Y., Yang, X.R., Fu, Z., Paudel, O., Abramowitz, J. and Birnbaumer, L. (2014) Classical Transient Receptor Potential 1 and 6 Contribute to Hypoxic Pulmonary Hypertension through Differential Regulation of Pulmonary Vascular Functions. Hypertension, 63, 173-180. http://dx.doi.org/10.1161/HYPERTENSIONAHA.113.01902

[30] Zhang, Y., Lu, W., Yang, K., Xu, L., Lai, N. and Tian, L. (2013) Bone Morphogenetic Protein 2 Decreases TRPC Expression, Store-Operated $\mathrm{Ca}^{2+}$ Entry, and Basal $\left[\mathrm{Ca}^{2+}\right]_{\mathrm{i}}$ in Rat Distal Pulmonary Arterial Smooth Muscle Cells. AJP: Cell Physiology, 304, C833-C843. http://dx.doi.org/10.1152/ajpcell.00036.2012

[31] Lin, M.J., Leung, G.P., Zhang, W.M., Yang, X.R., Yip, K.P. and Tse, C.M. (2004) Chronic Hypoxia-Induced Upregulation of Store-Operated and Receptor-Operated $\mathrm{Ca}^{2+}$ Channels in Pulmonary Arterial Smooth Muscle Cells: A Novel Mechanism of Hypoxic Pulmonary Hypertension. Circulation Research, 95, 496-505. http://dx.doi.org/10.1161/01.RES.0000138952.16382.ad

[32] Xu, L., Chen, Y., Yang, K., Wang, Y., Tian, L., Zhang, J., et al. (2014) Chronic Hypoxia Increases TRPC6 Expression and Basal Intracellular $\mathrm{Ca}^{2+}$ Concentration in Rat Distal Pulmonary Venous Smooth Muscle. PLoS ONE, 9, e112007. http://dx.doi.org/10.1371/journal.pone.0112007

[33] Wang, X.C., Li, M.X., Dang, X.Y., Li, P., Peng, Z. and Gao, Y.X. (2013) Calcineurin and TRPC6 Are Involved in ET-1-Induced Proliferation of Pulmonary Artery Smooth Muscle Cells. Acta Universitatis Medicinalis Nanjing (Natural Science), 33, 1191-1195.

[34] Liu, D., Yang, D., He, H., Chen, X., Cao, T. and Feng, X. (2009) Increased Transient Receptor Potential Canonical 
Type 3 Channels in Vasculature from Hypertensive Rats. Hypertension, 53, 70-76. http://dx.doi.org/10.1161/HYPERTENSIONAHA.108.116947

[35] Marvar, P.J., Vinh, A., Thabet, S., Lob, H.E., Geem, D., Ressler, K.J. and Harrison, D.G. (2012) T Lymphocytes and Vascular Inflammation Contribute to Stress-Dependent Hypertension. Biological Psychiatry, 71, 774-782. http://dx.doi.org/10.1016/j.biopsych.2012.01.017

[36] Thilo, F., Scholze, A., Liu, D.Y., Zidek, W. and Tepel, M. (2008) Association of Transient Receptor Potential Canonical Type 3 (TRPC3) Channel Transcripts with Proinflammatory Cytokines. Archives of Biochemistry and Biophysics, 471, 57-62. http://dx.doi.org/10.1016/j.abb.2007.12.006

[37] Wuensch, T., Thilo, F., Krueger, K., Scholze, A., Ristow, M. and Tepel, M. (2010) High Glucose-Induced Oxidative Stress Increases Transient Receptor Potential Channel Expression in Human Monocytes. Diabetes, 59, 844-849. http://dx.doi.org/10.2337/db09-1100

[38] Sanchez, M.E., Ibarra, S.A. and Gonzalez, E.C. (2010) Fyn Kinase Controls FceRI Receptor-Operated Calcium Entry Necessary for Full Degranulation in Mast Cells. Biochemical and Biophysical Research Communications, 391, 17141720. http://dx.doi.org/10.1016/j.bbrc.2009.12.139

[39] Vig, M. and Kinet, J.-P. (2009) Calcium Signaling in Immune Cells. Nature Immunology, 10, 21-27. http://dx.doi.org/10.1038/ni.f.220

[40] Harteneck, C. and Gollasch, M. (2011) Pharmacological Modulation of Diacylglycerol-Sensitive TRPC3/6/7 Channels. Current Pharmaceutical Biotechnology, 12, 35-41. http://dx.doi.org/10.2174/138920111793937943

[41] Kim, M.S., Lee, K.P., Yang, D., Shin, D.M., Abramowitz, J., Kiyonaka, S., et al. (2011) Genetic and Pharmacologic Inhibition of the $\mathrm{Ca}^{2+}$ Influx Channel TRPC3 Protects Secretory Epithelia from $\mathrm{Ca}^{2+}$-Dependent Toxicity. Gastroenterology, 140, 2107-2115. http://dx.doi.org/10.1053/j.gastro.2011.02.052

[42] Seo, K., Rainer, P.P., Shalkey, H.V., Lee, D.I., Jo, S.H. and Andersen, A. (2014) Combined TRPC3 and TRPC6 Blockade by Selective Small-Molecule or Genetic Deletion Inhibits Pathological Cardiac Hypertrophy. Proceedings of the National Academy of Sciences of the United States of America, 111, 1551-1556. http://www.ncbi.nlm.nih.gov/pubmed/24453217

[43] Koitabashi, N., Aiba, T., Hesketh, G.G., Rowell, J., Zhang, M., Takimoto, E., et al. (2009) Cyclic GMP/PKG-Dependent Inhibition of TRPC6 Channel Activity and Expression Negatively Regulates Cardiomyocyte NFAT Activation Novel Mechanism of Cardiac Stress Modulation by PDE5 Inhibition. Journal of Molecular and Cellular Cardiology, 48, 713724. http://dx.doi.org/10.1016/j.yjmcc.2009.11.015

[44] Kiso, H., Ohba, T., Iino, K., Sato, K., Terata, Y. and Murakami, M. (2013) Sildenafil Prevents the Up-Regulation of Transient Receptor Potential Canonical Channels in the Development of Cardiomyocyte Hypertrophy. Biochemical and Biophysical Research Communications, 436, 514-518. http://dx.doi.org/10.1016/j.bbrc.2013.06.002

[45] Wang, J., Yang, K., Xu, L., Zhang, Y., Lai, N. and Jiang, H. (2013) Sildenafil Inhibits Hypoxia-Induced Transient Receptor Potential Canonical Protein Expression in Pulmonary Arterial Smooth Muscle via cGMP-PKG-PPAR Axis. American Journal of Respiratory Cell and Molecular Biology, 49, 231-240. http://dx.doi.org/10.1165/rcmb.2012-01850C

[46] Liao, X.Y., Chen, M. and Yu, D.M. (2011) Effect of Anti-Hypertensive Medication on Expression of TRPC3 and TRPC6 in the Aorta of Spontaneous Hypertensive Rats. Chinese Circulation Journal, 26, 65-68.

[47] Yu, D.M., Chen, M. and Liao, X.Y. (2011) Effects of Antihypertensive Drugs on Expression of TRPC3 and TRPC6 in Left Ventricle of Spontaneously Hypertensive rats. International Journal of Cardiology, 152, S53. http://dx.doi.org/10.1016/j.ijcard.2011.08.640

[48] Toth, P., Tucsek, Z., Sosnowska, D., Gautam, T., Mitschelen, M. and Tarantini, S. (2013) Age-Related Autoregulatory Dysfunction and Cerebromicrovascular Injury in Mice with Angiotensin II-Induced Hypertension. Journal of Cerebral Blood Flow \& Metabolism, 33, 1732-1742. http://dx.doi.org/10.1038/jcbfm.2013.143

[49] Naoya, O., Motohiro, N., Ryuji, I., Kobayashi, H., Sumimoto, H. and Sato, Y. (2006) TRPC3 and TRPC6 Are Essential for Angiotensin II-Induced Cardiac Hypertrophy. The EMBO Journal, 25, 5305-5316. http://dx.doi.org/10.1038/sj.emboj.7601417

[50] Nijenhuis, T., Sloan, A.J., Hoenderop, J.G., Flesche, J., Goor, H. and Kistler, A.D. (2011) Angiotensin II Contributes to Podocyte Injury by Increasing TRPC6 Expression via an NFAT-Mediated Positive Feed-Back Signaling Pathway. The American Journal of Pathology, 179, 1719-1732. http://dx.doi.org/10.1016/j.ajpath.2011.06.033 


\section{Glossary}

\begin{tabular}{|c|c|}
\hline Abbreviations & Full Name \\
\hline $\mathrm{EH}$ & essential hypertension \\
\hline TRPC3 & transient receptor potential canonical 3 \\
\hline TRPC6 & transient receptor potential canonical 6 \\
\hline PLC & phospholipase C \\
\hline VDCC & voltage dependent calcium channel \\
\hline ROCC & receptor operated calcium channel \\
\hline SOCC & store-operated calcium channel \\
\hline DAG & diacylglycerol \\
\hline NCX & $\mathrm{Na}^{+} / \mathrm{Ca}^{2+}$ exchanger \\
\hline CaSR & calcium sensing receptor \\
\hline SHR & spontaneous hypertension rat \\
\hline PIP2 & phosphatidylinositol-4, 5-bisphosphate \\
\hline SMC & smooth muscle cell \\
\hline NFAT & nuclear factor of activated $\mathrm{T}$ cells \\
\hline HIF-1 & hypoxia-inducible factor 1 \\
\hline Ang II & angiotensin II \\
\hline ET-1 & endothelin-1 \\
\hline PDE 5 & phosphodiesterase type 5 \\
\hline PAH & pulmonary arterial hypertension \\
\hline PKG & protein kinase $\mathrm{G}$ \\
\hline ACEI & angiotensin converting enzyme inhibitor \\
\hline ARB & angiotensin receptor blocker \\
\hline СCB & calcium channel blocker \\
\hline L-VDCC & L-type voltage dependent calcium channel \\
\hline
\end{tabular}

